\title{
Label-free structural photoacoustic tomography of intact mouse brain
}

Lei Li, Jun Xia, Guo Li, Alejandro Garcia-Uribe, Lihong V. Wang

Lei Li, Jun Xia, Guo Li, Alejandro Garcia-Uribe, Lihong V. Wang, "Label-free structural photoacoustic tomography of intact mouse brain," Proc. SPIE 9323, Photons Plus Ultrasound: Imaging and Sensing 2015, 93230M (11 March 2015); doi: $10.1117 / 12.2077236$

SPIE. Event: SPIE BiOS, 2015, San Francisco, California, United States 


\title{
Label-free structural photoacoustic tomography of intact mouse brain
}

\author{
Lei $\mathrm{Li}^{\mathrm{ab}}$, Jun $\mathrm{Xia}^{\mathrm{a}}$, Guo Li ${ }^{\mathrm{a}}$, Alejandro Garcia-Uribe ${ }^{\mathrm{a}}$, Lihong V. Wang*ba \\ ${ }^{a}$ Department of Electrical and System Engineering, Washington University in St. Louis \\ One Brookings Dr., St. Louis, MO, 63130; \\ ${ }^{\mathrm{b}}$ Optical Imaging Laboratory, Department of Biomedical Engineering, Washington University in St. \\ Louis, One Brookings Dr., St. Louis, MO, 63130
}

\begin{abstract}
Capitalizing on endogenous hemoglobin contrast, photoacoustic computed tomography (PACT), a deep-tissue highresolution imaging modality, has drawn increasing interest in neuro-imaging. However, most existing studies are limited to functional imaging on the cortical surface, and the deep-brain structural imaging capability of PACT has never been demonstrated. Here, we explicitly studied the limiting factors of deep-brain PACT imaging. We found that the skull distorted the acoustic signal and blood suppressed the structural contrast from other chromophores. When the two effects are mitigated, PACT can provide high-resolution label-free structural imaging through the entire mouse brain. With 100 $\mu \mathrm{m}$ in-plane resolution, we can clearly identify major structures of the brain, and the image quality is comparable to that of magnetic resonance microscopy. Spectral PACT studies indicate that structural contrasts mainly originate from cytochrome and lipid. The feasibility of imaging the structure of the brain in vivo has also been discussed. Our results demonstrate that PACT is a promising modality for both structural and functional brain imaging.
\end{abstract}

Keywords: label-free imaging, structural photoacoustic tomography, brain imaging, photoacoustic computed tomography,

\section{INTRODUCTION}

Revealing how the brain works is a grand challenge worth our every effort. It will provide the key to understanding and treating neurological diseases [1]. Over the last few decades, various functional and structural brain imaging techniques have been developed, and some can image both structures and functions using the same modality. For instance, magnetic resonance imaging (MRI) using the T1 and T2 contrasts can image the structure of the brain over a large volume. Using the diffuse tensor (DT) method, it can also reveal fractional anisotropy, three dimensional (3D) diffusivity, and connection information. Functional MRI can also be achieved based on the blood-oxygen-level dependent (BOLD) contrast. However MRI works on time scales of minutes [2,3] and requires a costly magnetic field to achieve high spatial resolution $(50-100 \mu \mathrm{m})[4,5]$. Microscopic DT imaging requires additional tissue staining and takes more than one day for data acquisition [6-8]. Optical imaging techniques, such as confocal fluorescence microscopy and twophoton microscopy, are other powerful tools for both structural and functional brain imaging [9-12]. Various biomolecules, such as hemoglobin, cytochrome, melanin, and lipid [13-15], possess different optical properties, which provide contrasts for structural brain imaging. Using endogenous and exogenous contrasts, light can also be used to monitor functional dynamics, such as hemodynamics and neuron activities [10, 16, 17]. However, deep-tissue optical imaging at high spatial resolution is a major challenge because of tissue's strong optical scattering. Thus most optical imaging techniques can image only $1 \mathrm{~mm}$ deep into the brain [1].

Fortunately, by converting photons into ultrasonic waves, which are orders of magnitude less scattered than light, photoacoustic (PA) tomography (PAT) can form images by detecting the pressure waves induced by the thermoelastic expansion of light-absorbing objects $[18,19]$. PAT can break through the optical diffusion limit $(\sim 1 \mathrm{~mm}$ depth $)$ by capitalizing on the low acoustic scattering in tissue. Moreover, the scalability of PAT provides an unprecedented opportunity to bridge the gap between microscopic and macroscopic images. PAT is capable of anatomical, functional, molecular, and metabolic imaging of small animals, with highly scalable spatial resolution and penetration depth [2022]. Photoacoustic computed tomography (PACT), a major implementation of PAT, provides fast data acquisition, submillimeter resolution, and deep penetration beyond the optical diffusion limit [23-26]. Non-invasive, label-free, and functional PACT of the brains of small animals has been demonstrated by accurately mapping brain lesions and cerebral

Photons Plus Ultrasound: Imaging and Sensing 2015, edited by Alexander A. Oraevsky, Lihong V. Wang

Proc. of SPIE Vol. 9323, 93230M - (c) 2015 SPIE · CCC code: 1605-7422/15/\$18

doi: $10.1117 / 12.2077236$

Proc. of SPIE Vol. $932393230 \mathrm{M}-1$ 
hemodynamics [27]. Functional imaging of brain metabolism and hemodynamic changes also has been done by PACT $[28,29]$.

Here, for the first time, we experimentally demonstrate that PACT is able to provide label-free structural imaging of the whole mouse brain. The optical contrast yields images whose quality is comparable to that of magnetic resonance microscopy (MRM). With $100 \mu \mathrm{m}$ lateral resolution and $400 \mu \mathrm{m}$ elevational resolution, most deep brain anatomical structures have been clearly resolved through the entire brain. We also found by spectral analysis that the structural contrast comes mainly from cytochrome and lipid.

\section{MATERIALS AND METHODS}

\section{Full-ring photoacoustic computed tomography system}

Figure 1 is a schematic of the PACT system setup. To provide illumination in the visible band (from $420 \mathrm{~nm}$ to $680 \mathrm{~nm}$ ), an optical parametric oscillator (OPO) laser (BasiScan 120, Spectra-Physics) is pumped by an Nd:YAG laser (Brilliant $\mathrm{B}$, Quantel) with a third harmonic generator $(355 \mathrm{~nm})$. The laser pulse width is $6 \mathrm{~ns}$, and the pulse repetition rate is 10 Hz. The incident laser beam, reflected by a right-angle prism (PS912, Thorlabs), is homogenized through an engineered diffuser (EDC-5, RPC Photonics) to provide uniform illumination over the mouse brain. The maximum laser fluence on the scalp is approximately $6 \mathrm{~mJ} / \mathrm{cm} 2$, which is well below the American National Standards Institute safety limit (20 $\mathrm{mJ} / \mathrm{cm} 2$ in the visible spectral region). The photoacoustic signals are detected by a $5 \mathrm{~cm}$ diameter full-ring ultrasonic transducer array (Imasonic Inc.) with 512 elements, $5 \mathrm{MHz}$ central frequency, and more than $80 \%$ one-way bandwidth. Each element is cylindrically focused to produce an axial focal depth of $19 \mathrm{~mm}$. The combined foci of all elements form a uniform imaging region of $20 \mathrm{~mm}$ diameter and $1 \mathrm{~mm}$ thickness [9]. Within this region, the radial resolution is $100 \mu \mathrm{m}$ and the tangential resolution is $100-250 \mu \mathrm{m}$ [30]. The data acquisition system has 64 channels with 8-fold multiplexing. After a complete data acquisition from all 512 elements, the raw data are used to reconstruct an image based on the universal back-projection algorithm [31]. Three-dimensional (3D) images can be obtained by scanning the sample along the elevational direction. The imaging speed of the full-ring PACT system is $1.6 \mathrm{~s}$ per frame (per cross-section).

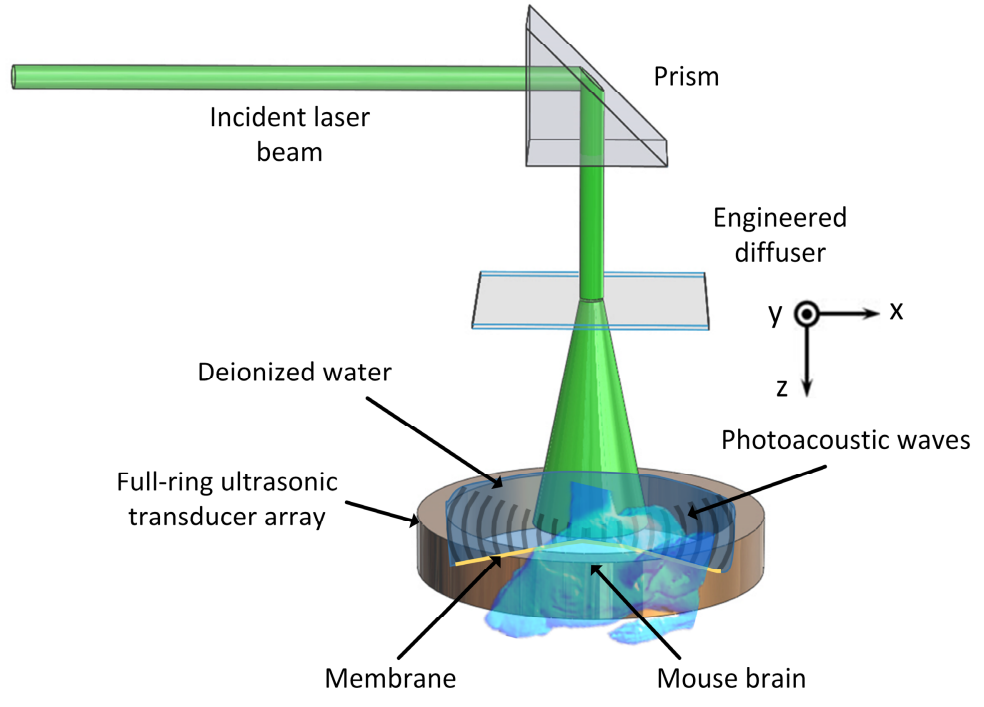

Figure 1. Schematic of full-ring photoacoustic computed tomography system.

\section{Label free photoacoustic imaging}

Based on the photoacoustic effect, if all absorbed optical energy is converted into heat, the initial pressure is given by [32]

$$
p_{0}=\Gamma \mu_{a} F
$$


where $F$ is the optical fluence $\left(\mathrm{J} / \mathrm{cm}^{2}\right), \mu_{\mathrm{a}}$ is the optical absorption coefficient $\left(\mathrm{cm}^{-1}\right)$, and $\Gamma$ is the Grueneisen parameter. It follows from Eq. (1) that PAT is exquisitely sensitive to optical absorption. In fact, among all optical imaging modalities, PAT has the highest sensitivity to optical absorption because it converts a small change in the optical absorption coefficient to an equal fractional change in the ultrasound signal, tantamount to a relative sensitivity of $100 \%$ [33]. In principle, PAT can be used to image any molecule, once the absorption band is found. For instance, PAT has been used to image oxy- and deoxy-hemoglobin [22, 34-37], melanin [38, 39], water [40-42], lipids [43-45], DNA and RNA [46-48], and cytochromes [49, 50].

Figure 2a indicates that hemoglobin is the most optically absorbing contrast for wavelengths below $1000 \mathrm{~nm}$. In the visible spectral region, its absorption coefficient can exceed that of other chromosphores by more than two orders of magnitude. While hemoglobin allows acquisition of stunning vascular images, it also obscures PA signals from other endogenous chromosphores, such as cytochromes and lipid. As shown in Fig. 2b, with $600 \mathrm{~nm}$ light illumination, a PACT image acquired at the brain surface shows rich cortical vasculature. Signals from major vessels, such the superior sagittal sinus, are so strong that their shadows even appear in the deep brain image (Fig. 2c). Nevertheless, the skull strongly attenuates and distorts the acoustic signal. Consequently, even though different brain structures have different cytochrome and lipid concentrations, which might provide us with structural contrast, we still cannot differentiate them in the deep brain image.

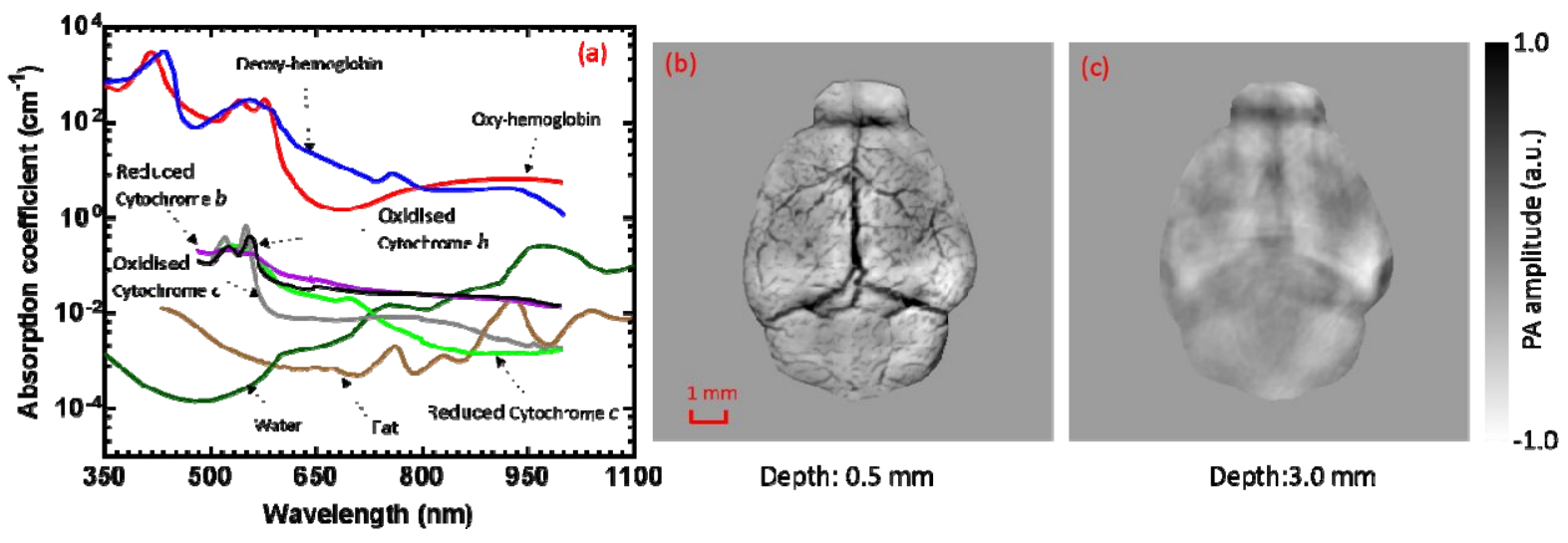

Figure 2. Label free photoacoustic imaging. (a) Absorption coefficient spectra of endogenous tissue chromophores at typical concentrations in the human body (Adapted from http://omlc.ogi.edu/spectra and

http://www.ucl.ac.uk/medphys/research/borl/intro/spectra) (b) In vivo imaging of the mouse brain cortex through the intact skull (imaging depth: $0.5 \mathrm{~mm}$ beneath the skull surface). (c) In vivo imaging of the deep mouse brain (imaging depth: $3.0 \mathrm{~mm}$ beneath the skull surface).

\section{Saline perfused mouse brain imaging by PACT}

Figure 2a indicates that if hemoglobin is removed from tissue, cytochrome becomes the dominant absorber in the visible region. In a mouse brain, by dry weight, gray matter has 55\% protein and 33\% lipid, white matter has $55 \%$ lipid and $40 \%$ protein, and myelin has $70 \%$ lipid and $29 \%$ protein [42]. Thus the difference in protein concentrations can be utilized to differentiate the brain structures. To remove the hemoglobin, we used saline perfusion.

The saline perfused mice were prepared by the Hope Center Animal Surgery Core at Washington University, following the standard mouse transcardial perfusion protocol. Blood was removed from the body tissues by pumping phosphate buffered saline into the left ventricle and draining the blood from the aorta, which was cut with scissors. After 4-5 minutes, the fluid exiting the aorta was clear and the liver had turned white. At this point most of the blood in the body had been replaced with saline. We then dissected the head and placed it into $10 \%$ paraformaldehyde (PFA) solution for preservation. After 24 hours of fixation in 10\% PFA, we embedded the head in 3\% agar gel for imaging. 


\section{RESULTS}

\section{Mouse brain structural imaging by PACT}

The saline perfused mouse head with skull intact was imaged with $600 \mathrm{~nm}$ laser illumination. Media 1a (Fig. 3a) shows cross-sectional images at different depths. Without the dominant hemoglobin absorption, this image looks totally different from Fig. 2b. However, we still cannot clearly identify the structure of the brain, possibly due to light attenuation from the top skull and acoustic distortion from the side skull bone. To study the two effects, we first removed the top skull. The resulting image (Fig. 3b) did not show much improvement, which indicates that light attenuation from the skull is negligible. We further removed the side skull bone and acquired another image, which shows different brain structures with astonishing clarity (Fig. 3c). To our knowledge, this is the first time that PAT has revealed deep structures of the brain in such detail based on endogenous contrast. Next, we removed the entire mouse brain from the rest of the skull and acquired a new image of the brain (Fig. 3d). Because the mouth and nasal cavities, which interfere with acoustic propagation, were removed, the image clarity was further improved.
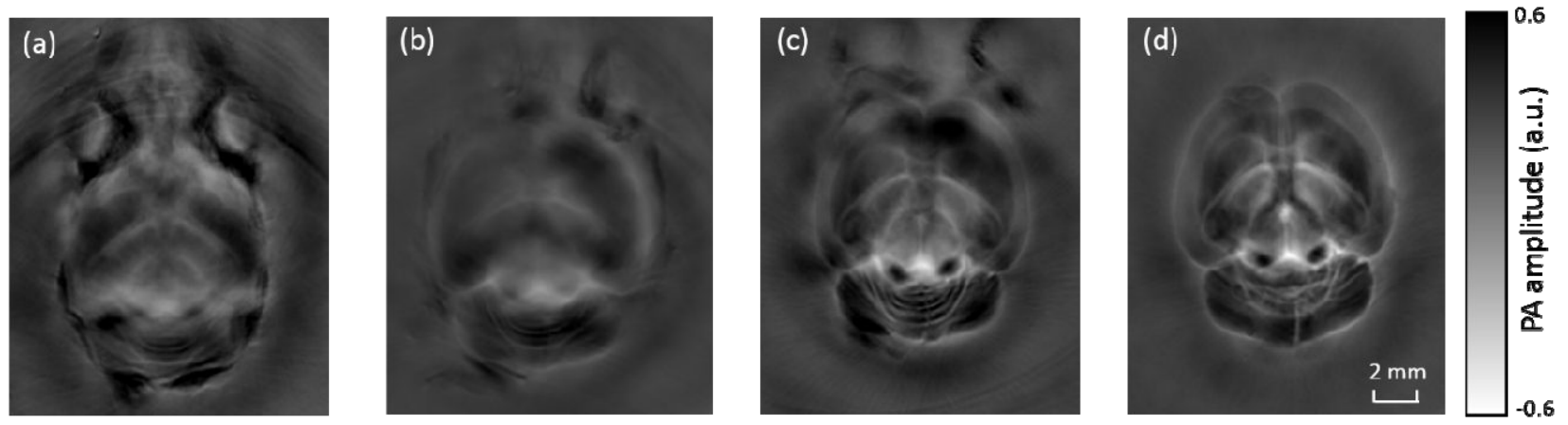

Figure 3. PACT of the saline perfused brain with and without the skull. (a) Image of in situ brain with skull intact (media 1a). (b) Image of brain with top skull removed. (c) Image of brain with top and side skull removed; (d) Image of brain with all of the skull removed. Media 1: http://dx.doi.org/10.1117/12.2077236.1
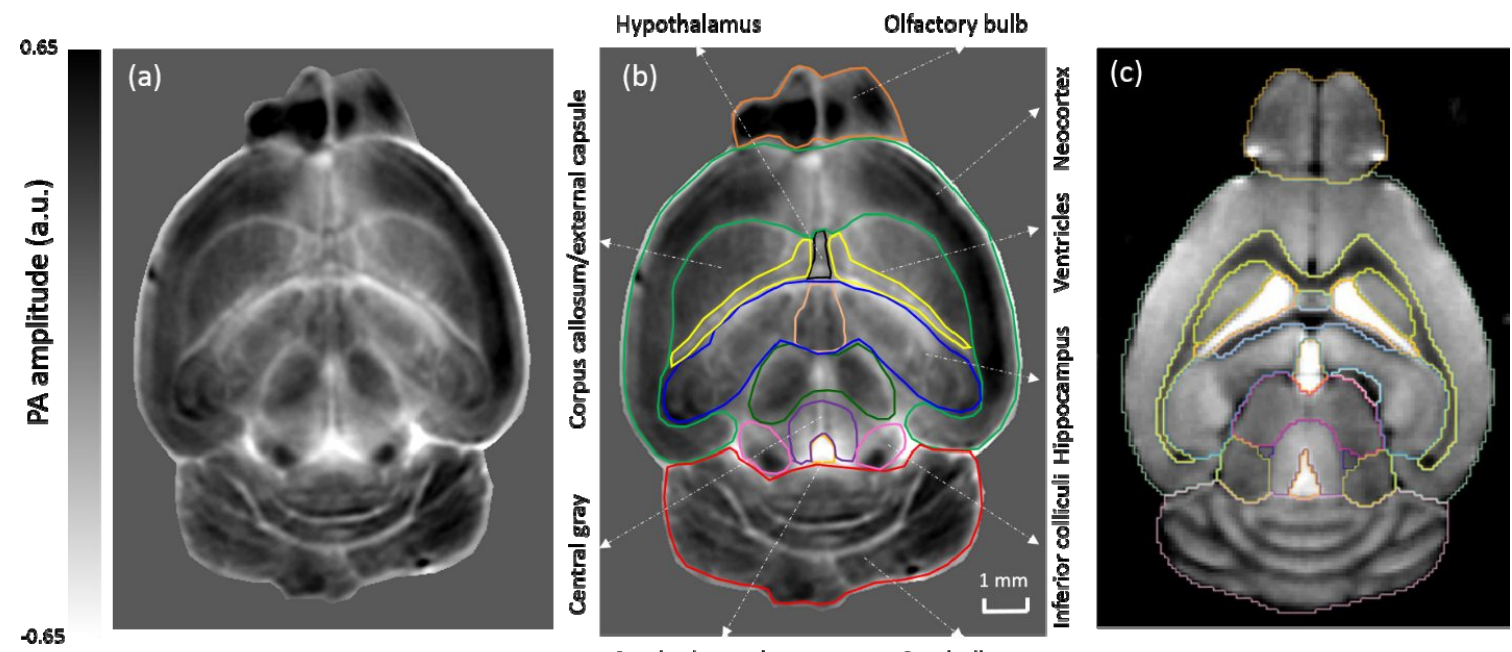

Cerebral aqueduct

Cerebellum

Figure 4. Validation of the PACT image of the saline perfused mouse brains without the skull. (a) Cross-sectional PACT image of the mouse brain at $2.8 \mathrm{~mm}$ depth, clearly resolving the structure of the brain. (b) Segmented and annotated image from (a). (c) One slice of a 3D high-resolution MRM image with its structural segmentation superimposed as colored lines, chosen as a gold standard for validation of PACT [51] (Courtesy of Frontiers in Neuroscience). 
Figure 4a shows a label-free PACT image (acquired $3.0 \mathrm{~mm}$ below the brain surface) of another saline-perfused mouse brain, where the olfactory bulb was preserved. Images at other depths are shown in media $2 \mathrm{a}$, from which we can see that PACT can image through the entire brain as deep as $5.0 \mathrm{~mm}$. To better illustrate different regions, we segmented Fig. 4a based on the PA amplitude, and labeled different segmentations. For comparison, we chose one slice of a 3D high-resolution MRM image, with its structural segmentation superimposed as colored lines [51]. The mouse brain PACT image shows a nearly perfect match with the MRM image. Different brain structures are clearly identified, including the central gray, cerebellum, cerebral aqueduct, corpus callosum, hippocampus, hypothalamus, inferior colliculus, neocortex, olfactory bulb, and ventricles. To our knowledge, this is the first time that PACT has clearly shown deep structures of the brain with rich contrast among different brain tissues, with the brain intact and without any labeling. Such deep brain structures have not been shown by other photoacoustic imaging techniques or optical imaging modalities.

\section{Spectral PACT imaging}

To find the optimal wavelength for structural imaging and to verify the origin of contrasts, we varied the laser wavelength from $480 \mathrm{~nm}$ to $680 \mathrm{~nm}$ with a $20 \mathrm{~nm}$ interval. Figure 5 shows the spectral PACT images of a mouse brain at one selected depth (around $2.8 \mathrm{~mm}$ below the brain surface), and media 3 shows cross-section images at varied depths for each wavelength used (from $480 \mathrm{~nm}$ to $680 \mathrm{~nm}$ ). From Fig. 2a, we see that cytochrome has strong absorption in the range of 480-500 $\mathrm{nm}$. The strong attenuation of this short wavelength light leads to shallow penetration, and thus the images at $480 \mathrm{~nm}$ and $500 \mathrm{~nm}$ have low contrast-to-noise ratios (CNRs) at depth. Longer wavelength (640 to $680 \mathrm{~nm}$ ) light suffers less attenuation, but the low absorption results in weak PA signals. Therefore, the images at $640-680 \mathrm{~nm}$ illumination have a lower CNR. A quantitative spectral analysis is discussed in detail in the following section.

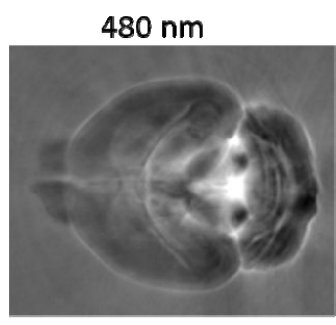

$500 \mathrm{~nm}$

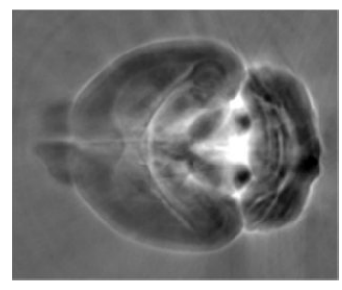

$520 \mathrm{~nm}$

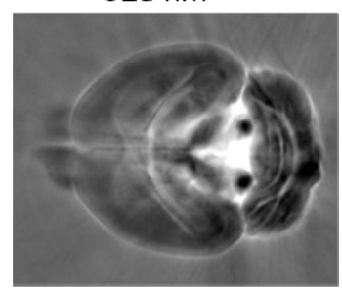

$540 \mathrm{~nm}$

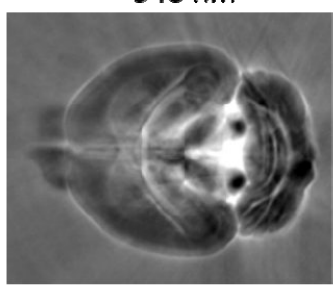

$560 \mathrm{~nm}$

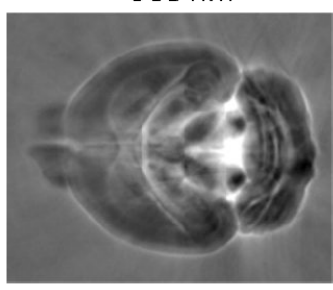

$580 \mathrm{~nm}$

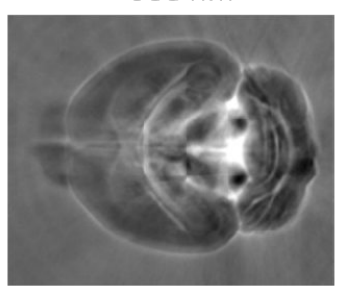

$590 \mathrm{~nm}$

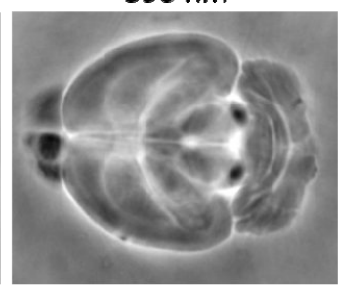

$600 \mathrm{~nm}$

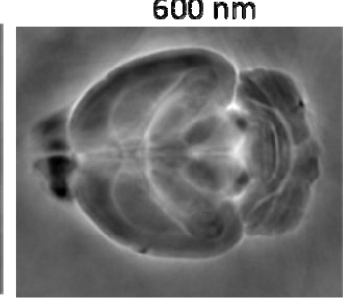

$620 \mathrm{~nm}$

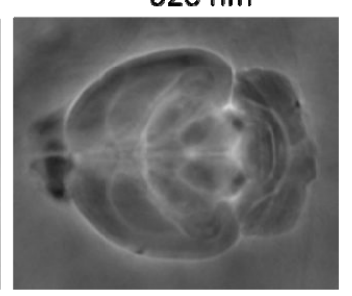

$640 \mathrm{~nm}$

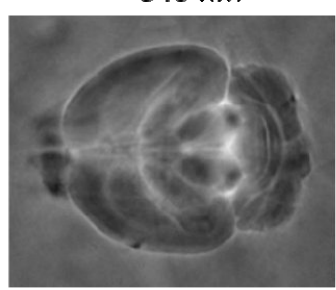

$660 \mathrm{~nm}$

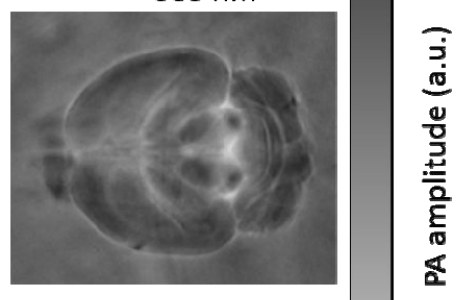

$680 \mathrm{~nm}$
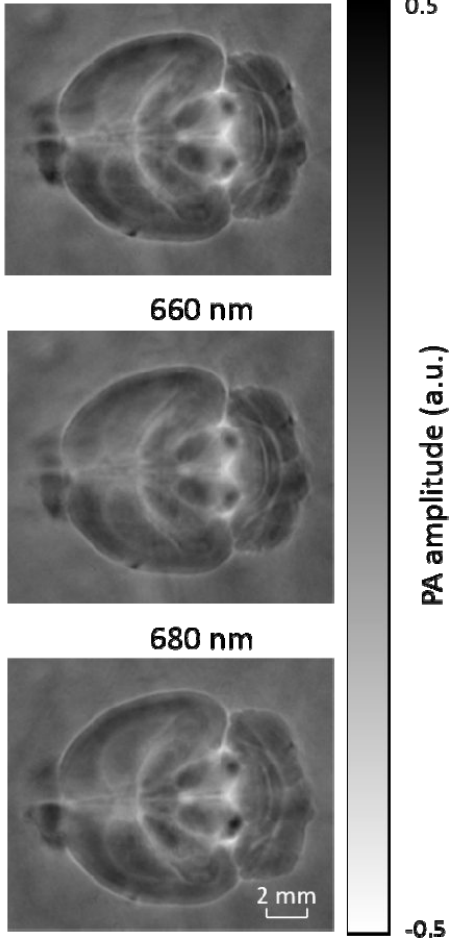

Figure. 5. Spectral PACT of a mouse brain at $2.8 \mathrm{~mm}$ depth.

Media 2: http://dx.doi.org/10.1117/12.2077236.2

Media 3: http://dx.doi.org/10.1117/12.2077236.3 


\section{DISCUSSION AND CONCLUSION}

After the removal of blood, the most probable chromophores contributing to the brain structure images are water, cytochrome, and lipid. Because the water contents in various brain tissues are very similar [52], water provides only a relatively constant background in PACT images. Thus the structural contrast should mainly come from cytochrome and lipid. Each pixel in a single-wavelength PACT image represents a combined contribution from $M$ optical absorbers with known molar extinction coefficient spectra $a_{m}$ and unknown concentrations $c_{m}(m=1,2, \cdots, M)$. Then the spectral decomposition equation can be expressed as

$$
\mu_{a}\left(\lambda_{n}\right)=\sum_{m=1}^{M} a_{m}\left(\lambda_{n}\right) c_{m}, n=1,2, \cdots, N
$$

where $\lambda_{n}$ is the nth wavelength used for PA excitation. Based on the known extinction coefficients, the concentrations of the optical absorbers can be quantified.
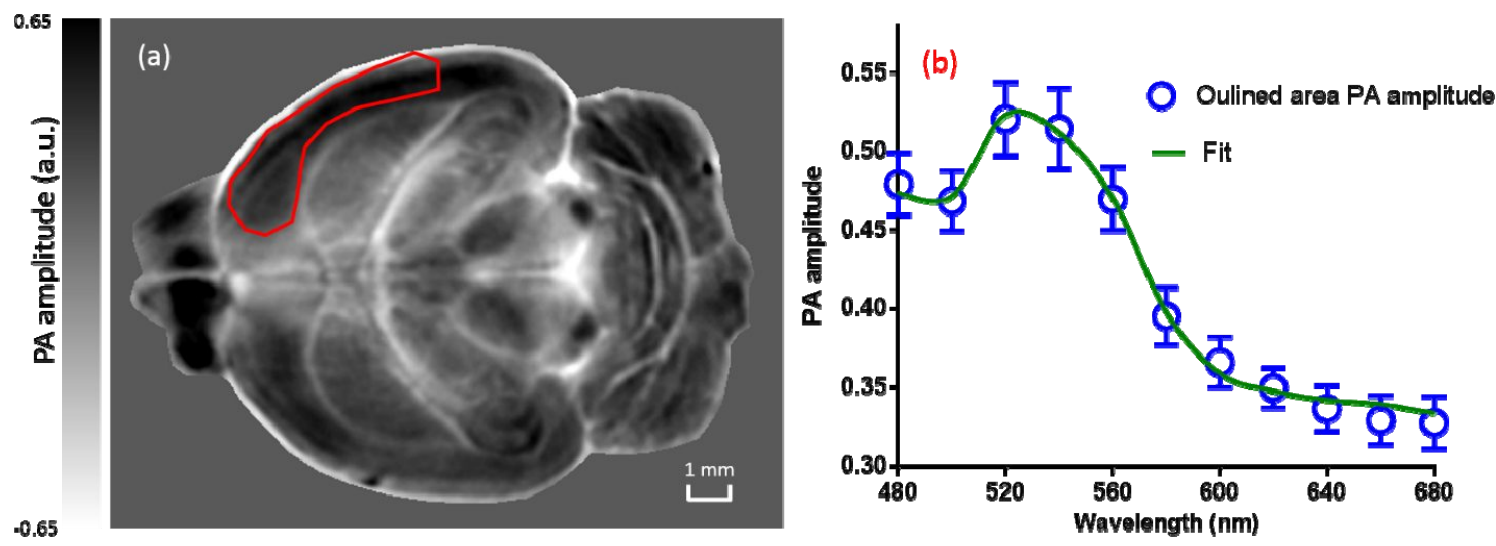

Figure 6. Spectral analysis of the origin of optical absorption in the brain. (a) Cross-section imaged at $600 \mathrm{~nm}$ wavelength at $2.8 \mathrm{~mm}$ depth as part of the $3 \mathrm{D}$ brain imaging, with red outlined areas segmented for analysis. (b)

Measured PA spectrum and least-squares fit with a mixture of cytochromes $b$ and $c$

In mammals, the brain performs numerous computationally intensive tasks, such as information processing, perception, motion control, and learning, and thus consumes a large amount of energy in proportion to its volume. Mitochondria, the energy source, populate the cytoplasm of mammalian cells, including neurons, which rely on mitochondrial energy production for survival [53]. The absorption sources of the mitochondria are mainly cytochromes $b$ and $c$ [49, 54]. However, myelin has a high concentration of lipid, which has orders of magnitude weaker absorption than that of cytochrome over the spectral range of $480 \sim 680 \mathrm{~nm}$. Therefore, the distributions of the different chromophores map the brain with sharp contrast in PACT images.

In the 3D brain PACT images, at $3.0 \mathrm{~mm}$ below the brain surface, the spectral PA responses (normalized by the laser fluence) of the neocortex area encircled by the red line are plotted in Fig. 6b (labeled by circles). The neocortex consists of gray matter, or neuronal cell bodies and unmyelinated fibers. The high concentration of mitochondria in neuronal cell bodies might be responsible for the optical absorption. Because the absorption sources in mitochondria are mainly cytochromes $b$ and $c[49,54]$, the PA spectrum of the red outlined area was fitted according to Eq. (2) with a mixture of $65 \pm 12 \%$ (molar ratio, mean \pm standard error) cytochrome $b$ and $35 \pm 9 \%$ cytochrome $c$, which is in agreement with the measured concentration of cytochrome in mitochondria [49]. The squared 2-norm of the residual is 0.003 . The accuracy of this result, however, is subject to the possible presence of other neglected absorbing proteins (such as cytochrome p450, nitric oxide synthases, and myeloperoxidase) with similar spectra. Absorption from other sources, such as water, flavoproteins, nicotinamide adenine dinucleotide, or other neglected hemeproteins, is orders of magnitude weaker than cytochrome, and was removed as a constant background during the fitting. 
To further confirm that structural contrast comes mainly from cytochrome and lipid, another area in the cerebellum (Fig. 7a) was segmented for spectral analysis. In a close-up image (Fig. 7b), white and black stripes correspond to cerebellar white matter and the granular layer, respectively $[55,56]$. The cerebellar white matter, made up largely of myelinated nerve fibers, has a high concentration of lipid, and the granular layer has a high concentration of cytochrome. We calculated the absorption ratio (AR) between the granular cell layer and the cerebellar white matter as follows:

$$
A R=\frac{\operatorname{mean}\left(P A_{g c l}\right)}{m e a n\left(P A_{c w m}\right)},
$$

Here $P A_{c u m}$ is the PA amplitude of the cerebellar white matter region, and $P A_{g c l}$ is the PA amplitude of the granular layer region. We compare the result with the AR of cytochrome to lipid, which is obtained by taking the ratio point by point between the cytochrome absorption spectrum and the lipid absorption spectrum shown in Fig. 2a. The two results match very well (Fig. 7c), which indicates that the contrast of the PACT image of the saline-perfused brain comes mainly from cytochrome and lipid.
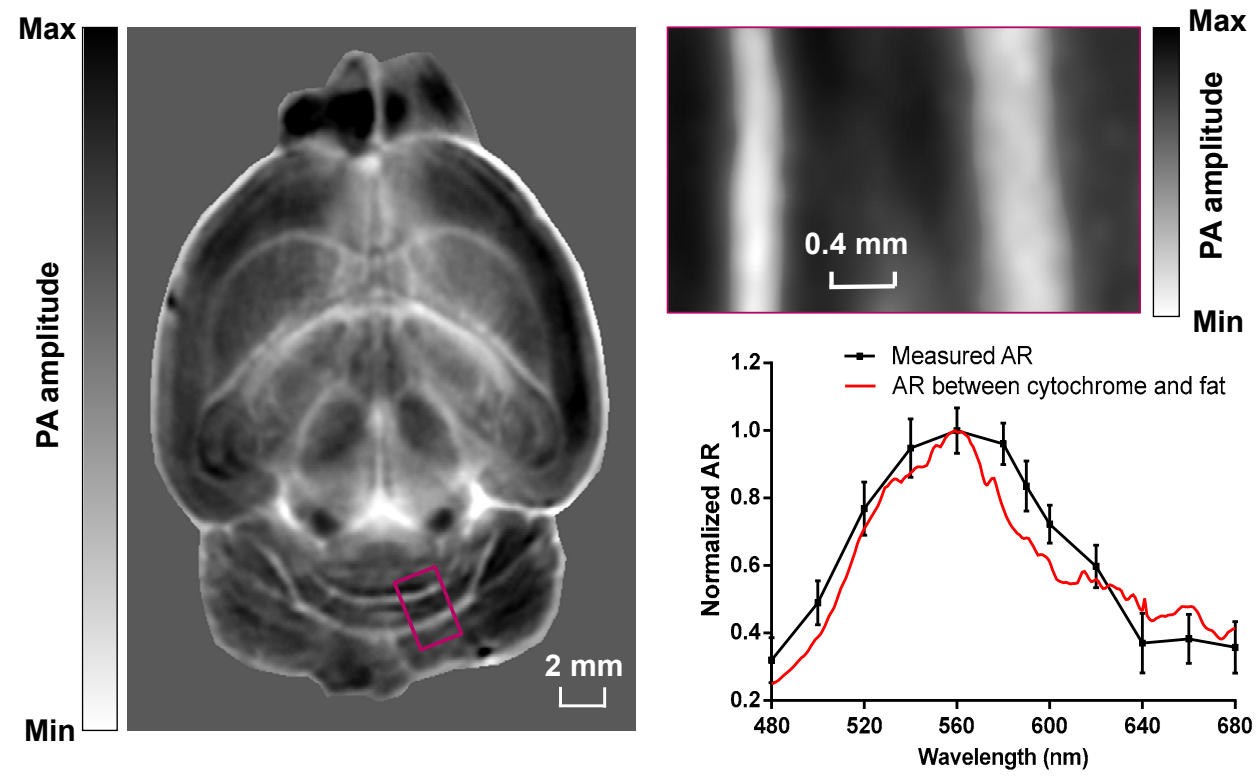

Figure 7. Analysis of the origins of the contrast. (a) Cross-section imaged at $600 \mathrm{~nm}$ wavelength at $2.8 \mathrm{~mm}$ depth selected from the 3D brain images, with brown outlined areas segmented for analysis. (b) Close-up of the region inside the brown square in (a). (c) Comparison of the measured AR between the granular cell layer and the cerebellar white matter and the AR between cytochrome and fat.

We also studied the feasibility of structural imaging on non-perfused brains. In order to minimize the distortion of PA waves due to bone, we removed the skull of a euthanized mouse, fully exposing the blood-intact brain. Images acquired at different depths are shown in Fig. 8. It can be seen that both of the cortex vessels (Fig. 8a) and deep brain structure (Figs. $8 \mathrm{~b}$ and $8 \mathrm{c}$ ) are clearly resolved. Most of the cerebral structures, such as the neocortex, corpus callosum, hippocampus, hypothalamus, and ventricles can be identified (Fig. 8c). Compared to Fig. 4a, though, the presence of hemoglobin decreases the structural contrast of the brain image, as shown in media 4:

http://dx.doi.org/10.1117/12.2077236.4 

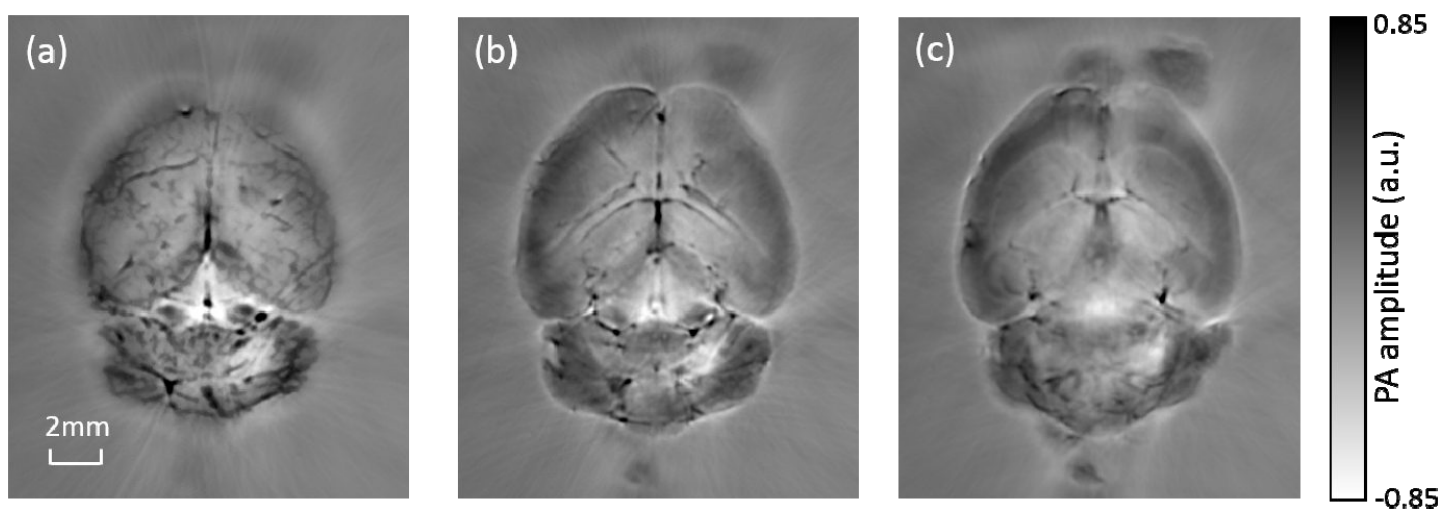

Figure 8. PACT image of a whole blood-perfused brain, showing both the vasculature and brain structure at depths of (a) $0.3 \mathrm{~mm}$, (b) $2.1 \mathrm{~mm}$, and (c) $2.9 \mathrm{~mm}$.

Nevertheless, the main hindrance to imaging the structure of the brain in vivo is the bone effect. Fig. 3(b) shows that removing just the top skull would not help us much. In fact, the top skull removal created irregular bone boundaries, which further distorted the acoustic wave. Compared to the top skull, the side skull is thicker and has more complex structures. Because of our signal detection geometry (Fig. 1), these side bones have a stronger effect on the PA signal. To address this issue, there are two possible approaches. First, we can thin the skull to mitigate the acoustic distortion. Top skull thinning is routinely applied in confocal or two-photon microscopy $[57,58]$. Thinning the side skull may require special attention to avoid loss of blood. Second, the acoustic properties of the head can be incorporated into PACT image reconstruction. This will require accurate measurement of the skull's geometry and acoustic properties, which can be achieved with other imaging modalities. For example, by using X-ray CT , the skull morphology and composition can be obtained and incorporated into a time-reversal-based reconstruction algorithm to correct for the skull distortion [59]. Ultrasound tomography (UST) has also been integrated into PACT to measure the acoustic properties, which were then used to inform the PACT image reconstruction [60]. With continuing advances in imaging techniques and reconstruction algorithms, we believe PACT will soon allow in vivo imaging of the deep brain in addition to the cortex.

In summary, we have experimentally demonstrated, for the first time, that PACT is able to provide label-free imaging of whole mouse brains, with MRM image quality. As a quantitative imaging modality, PACT can spectrally differentiate different chromophores (cytochromes and lipid) in brain tissue by virtue of its optical absorption contrast. As a deep imaging modality, PACT can resolve deep structures of the brain owing to its high ultrasonic resolution. With these merits, we expect PACT to be applied to more brain structural and functional studies in the future.

\section{ACKNOWLEDGMENT}

The authors appreciate Prof. James Ballard's close reading of the manuscript, and thank Ernie Gonzales, Dr. Junjie Yao, Dr. Chi Zhang, and Chiye Li for their useful discussions and technical assistance. This work was sponsored in part by National Institutes of Health (NIH) grants DP1 EB016986 (NIH Director's Pioneer Award), R01 CA186567 (NIH Director's Transformative Research Award), R01 EB016963, R01 EB010049, and R01 CA159959. L. V. Wang has a financial interest in Microphotoacoustics, Inc., and Endra, Inc., which, however, did not support this work. 


\section{REFERENCES}

[1] J. D. Pollock, "Deep imaging technology needed for NIH BRAIN initiative," Journal of Biomedical Optics, 19(3), 030601-030601 (2014).

[2] N. Chuang, S. Mori, A. Yamamoto et al., "An MRI-based atlas and database of the developing mouse brain," Neuroimage, 54(1), 80-89 (2011).

[3] F. Hyder, K. L. Behar, M. A. Martin et al., "Dynamic magnetic-resonance-imaging of the rat-brain during forepaw stimulation," Journal of Cerebral Blood Flow and Metabolism, 14(4), 649-655 (1994).

[4] G. Nair, and T. Q. Duong, "Echo-planar BOLD fMRI of mice on a narrow-bore 9.4 T magnet," Magnetic Resonance in Medicine, 52(2), 430-434 (2004).

[5] X. P. Hu, and D. G. Norris, "Advances in high-field magnetic resonance imaging," Annual Review of Biomedical Engineering, 6, 157-184 (2004).

[6] D. Wu, J. D. Xu, M. T. McMahon et al., "In vivo high-resolution diffusion tensor imaging of the mouse brain," Neuroimage, 83, 18-26 (2013).

[7] Y. Jiang, and G. A. Johnson, "Microscopic diffusion tensor atlas of the mouse brain," Neuroimage, 56(3), 1235-1243 (2011).

[8] Y. Jiang, and G. A. Johnson, "Microscopic diffusion tensor imaging of the mouse brain," Neuroimage, 50(2), 465-471 (2010).

[9] V. Gradinaru, M. Mogri, K. R. Thompson et al., "Optical Deconstruction of Parkinsonian Neural Circuitry," Science, 324(5925), 354-359 (2009).

[10] F. Zhang, L. P. Wang, M. Brauner et al., "Multimodal fast optical interrogation of neural circuitry," Nature, 446(7136), 633-U4 (2007).

[11] E. S. Boyden, F. Zhang, E. Bamberg et al., "Millisecond-timescale, genetically targeted optical control of neural activity," Nature Neuroscience, 8(9), 1263-1268 (2005).

[12] R. Yuste, and W. Denk, "Dendritic spines as basic functional units of neuronal integration," Nature, 375(6533), 682-684 (1995).

[13] T. E. Matthews, J. W. Wilson, S. Degan et al., "In vivo and ex vivo epi-mode pump-probe imaging of melanin and microvasculature," Biomedical Optics Express, 2(6), 1576-1583 (2011).

[14] C. W. Freudiger, W. Min, B. G. Saar et al., "Label-Free Biomedical Imaging with High Sensitivity by Stimulated Raman Scattering Microscopy,” Science, 322(5909), 1857-1861 (2008).

[15] L. Cai, N. Friedman, and X. S. Xie, "Stochastic protein expression in individual cells at the single molecule level," Nature, 440(7082), 358-362 (2006).

[16] A. Y. Shih, J. D. Driscoll, P. J. Drew et al., "Two-photon microscopy as a tool to study blood flow and neurovascular coupling in the rodent brain," Journal of Cerebral Blood Flow and Metabolism, 32(7), 1277-1309 (2012).

[17] H. P. Lu, L. Y. Xun, and X. S. Xie, "Single-molecule enzymatic dynamics," Science, 282(5395), 1877-1882 (1998).

[18] J. Xia, J. J. Yao, and L. H. V. Wang, "Photoacoustic tomography: principles and advances (invited review)," Progress In Electromagnetics Research, 147, 22 (2014).

[19] L. H. V. Wang, and S. Hu, "Photoacoustic Tomography: In Vivo Imaging from Organelles to Organs," Science, 335(6075), 1458-1462 (2012).

[20] L. V. Wang, "Multiscale photoacoustic microscopy and computed tomography," Nature Photonics, 3(9), 503509 (2009).

[21] K. Maslov, H. F. Zhang, S. Hu et al., "Optical-resolution photoacoustic microscopy for in vivo imaging of single capillaries," Optics Letters, 33(9), 929-931 (2008).

[22] H. F. Zhang, K. Maslov, G. Stoica et al., "Functional photoacoustic microscopy for high-resolution and noninvasive in vivo imaging," Nature Biotechnology, 24(7), 848-851 (2006).

[23] J. Xia, M. R. Chatni, K. Maslov et al., "Whole-body ring-shaped confocal photoacoustic computed tomography of small animals in vivo," Journal of Biomedical Optics, 17(5), 050506 (3 pp.)-050506 (3 pp.) (2012).

[24] C. Li, A. Aguirre, J. Gamelin et al., "Real-time photoacoustic tomography of cortical hemodynamics in small animals," Journal of Biomedical Optics, 15(1), 010509 (3 pp.)-010509 (3 pp.) (2010).

[25] D. Razansky, M. Distel, C. Vinegoni et al., "Multispectral opto-acoustic tomography of deep-seated fluorescent proteins in vivo," Nature Photonics, 3(7), $412-417$ (2009). 
[26] M.-L. Li, O. Jung-Taek, X. Xie et al., "Simultaneous molecular and hypoxia imaging of brain tumors in vivo using spectroscopic photoacoustic tomography," Proceedings of the IEEE, 96(3), 481-489 (2008).

[27] X. D. Wang, Y. J. Pang, G. Ku et al., "Noninvasive laser-induced photoacoustic tomography for structural and functional in vivo imaging of the brain," Nature Biotechnology, 21(7), 803-806 (2003).

[28] J. J. Yao, J. Xia, K. I. Maslov et al., "Noninvasive photoacoustic computed tomography of mouse brain metabolism in vivo," Neuroimage, 64, 257-266 (2013).

[29] L.-D. Liao, M.-L. Li, H.-Y. Lai et al., "Imaging brain hemodynamic changes during rat forepaw electrical stimulation using functional photoacoustic microscopy," Neuroimage, 52(2), 562-570 (2010).

[30] J. Xia, Z. J. Guo, K. Maslov et al., "Three-dimensional photoacoustic tomography based on the focal-line concept," Journal of Biomedical Optics, 16(9), 3 (2011).

[31] M. H. Xu, and L. H. V. Wang, "Universal back-projection algorithm for photoacoustic computed tomography," Physical Review E, 71(1), 7 (2005).

[32] L. V. Wang, "Photoacoustic tomography," Scholarpedia, 9(2), (2014).

[33] L. V. Wang, "Tutorial on photoacoustic microscopy and computed tomography," Ieee Journal of Selected Topics in Quantum Electronics, 14(1), 171-179 (2008).

[34] L. Li, C. H. Yeh, S. Hu et al., "Fully motorized optical-resolution photoacoustic microscopy," Optics Letters, 39(7), 2117-2120 (2014).

[35] A. Ray, J. R. Rajian, Y. E. Lee et al., "Lifetime-based photoacoustic oxygen sensing in vivo," J Biomed Opt, 17(5), 057004 (2012).

[36] Y. Jiang, A. Forbrich, T. Harrison et al., "Blood oxygen flux estimation with a combined photoacoustic and high-frequency ultrasound microscopy system: a phantom study,” J Biomed Opt, 17(3), 036012 (2012).

[37] P. Beard, "Biomedical photoacoustic imaging," Interface Focus, 1(4), 602-631 (2011).

[38] J. A. Viator, J. Komadina, L. O. Svaasand et al., "A comparative study of photoacoustic and reflectance methods for determination of epidermal melanin content," Journal of Investigative Dermatology, 122(6), 14321439 (2004).

[39] S. E. Forest, and J. D. Simon, "Wavelength-dependent photoacoustic calorimetry study of melanin," Photochemistry and Photobiology, 68(3), 296-298 (1998).

[40] Z. Xu, Q. Zhu, and L. V. Wang, "In vivo photoacoustic tomography of mouse cerebral edema induced by cold injury," Journal of Biomedical Optics, 16(6), (2011).

[41] Z. Xu, C. Li, and L. V. Wang, "Photoacoustic tomography of water in phantoms and tissue," Journal of Biomedical Optics, 15(3), 036019 (6 pp.)-036019 (6 pp.) (2010).

[42] G. S. Scott Brady, R. Wayne Albers, Donald Price, [Basic Neurochemistry: Molecular, Cellular and Medical Aspects] Elsevier Academic Press, Burlington, MA 01803, USA(2006).

[43] P. Wang, P. Wang, H.-W. Wang et al., "Mapping lipid and collagen by multispectral photoacoustic imaging of chemical bond vibration," Journal of Biomedical Optics, 17(9), (2012).

[44] B. Wang, A. Karpiouk, D. Yeager et al., "Intravascular photoacoustic imaging of lipid in atherosclerotic plaques in the presence of luminal blood," Optics Letters, 37(7), 1244-1246 (2012).

[45] T. J. Allen, A. Hall, A. P. Dhillon et al., "Spectroscopic photoacoustic imaging of lipid-rich plaques in the human aorta in the 740 to $1400 \mathrm{~nm}$ wavelength range," Journal of Biomedical Optics, 17(6), (2012).

[46] D. K. Yao, K. Maslov, K. K. Shung et al., "In vivo label-free photoacoustic microscopy of cell nuclei by excitation of DNA and RNA," Optics Letters, 35(24), 4139-4141 (2010).

[47] M. R. Bugs, and M. L. Cornelio, "A new biophysics approach using photoacoustic spectroscopy to study the DNA-ethidium bromide interaction," European Biophysics Journal with Biophysics Letters, 31(3), 232-240 (2002).

[48] M. R. Bugs, and M. L. Cornelio, "Analysis of the ethidium bromide bound to DNA by photoacoustic and FTIR spectroscopy," Photochemistry and Photobiology, 74(4), 512-520 (2001).

[49] C. Zhang, Y. S. Zhang, D. K. Yao et al., "Label-free photoacoustic microscopy of cytochromes," J Biomed Opt, 18(2), 20504 (2013).

[50] C. Di Primo, E. Deprez, S. G. Sligar et al., "Origin of the photoacoustic signal in cytochrome p-450(cam): Role of the Arg186-Asp251-Lys178 bifurcated salt bridge," Biochemistry, 36(1), 112-118 (1997).

[51] Y. Ma, D. Smith, P. R. Hof et al., "In vivo 3D digital atlas database of the adult C57BL/6J mouse brain by magnetic resonance microscopy," Frontiers in Neuroanatomy, 2, 10 (2008). 
[52] W. L. Lin, R. Venkatesan, K. Gurleyik et al., "An absolute measurement of brain water content using magnetic resonance imaging in two focal cerebral ischemic rat models," Journal of Cerebral Blood Flow and Metabolism, 20(1), 37-44 (2000).

[53] M. Picard, and B. S. McEwen, "Mitochondria impact brain function and cognition," Proceedings of the National Academy of Sciences of the United States of America, 111(1), 7-8 (2014).

[54] A. V. Brusnichkin, D. A. Nedosekin, E. I. Galanzha et al., "Ultrasensitive label-free photothermal imaging, spectral identification, and quantification of cytochrome c in mitochondria, live cells, and solutions," Journal of Biophotonics, 3(12), 791-806 (2010).

[55] S. Kim, S. Pickup, O. Hsu et al., "Enhanced delineation of white matter structures of the fixed mouse brain using Gd-DTPA in microscopic MRI," Nmr in Biomedicine, 22(3), 303-309 (2009).

[56] M. Tanaka, K. Yamaguchi, T. Tatsukawa et al., "Connexin43 and bergmann glial gap junctions in cerebellar function," Frontiers in neuroscience, 2(2), 225-33 (2008).

[57] G. Yang, F. Pan, C. N. Parkhurst et al., "Thinned-skull cranial window technique for long-term imaging of the cortex in live mice," Nature Protocols, 5(2), 201-208 (2010).

[58] D. F. Marker, M.-E. Tremblay, S.-M. Lu et al., "A thin-skull window technique for chronic two-photon in vivo imaging of murine microglia in models of neuroinflammation," Journal of visualized experiments : JoVE(43), (2010).

[59] C. Huang, L. M. Nie, R. W. Schoonover et al., "Aberration correction for transcranial photoacoustic tomography of primates employing adjunct image data," Journal of Biomedical Optics, 17(6), 8 (2012).

[60] J. Xia, C. Huang, K. Maslov et al., "Enhancement of photoacoustic tomography by ultrasonic computed tomography based on optical excitation of elements of a full-ring transducer array," Optics Letters, 38(16), 3140-3143 (2013). 